\title{
Design and Validation of Affective Warning Pictorial on Cigarette Labels
}

\author{
Pat-Arin Chanduen and Arisara Jiamsanguanwong \\ Department of Industrial Engineering, Faculty of Engineering, Chulalongkorn University, Bangkok, Thailand
}

\begin{abstract}
The purpose of present study were to design and validate affective warning pictorials for cigarette label in Thailand. Brainstorming and survey techniques were used to collect the idea of possible warning pictorials. All ideas were grouped for finding candidated pictorials. Then, primary sixty warning pictorials were collected and equally classified into three affective warning pictorial groups as positive, neutral, and negative. Sixty Thai male engineering students participated in affective validation of warning pictorials using SAM rating. The International Affective Picture System (IAPS) was used to manipulate the affective state of participants to neutral affective state before the experiments. The results revealed that all affective warning pictorials were successfully evoked target affective states on participants. After refining, thirty affective warning pictorials were provided as positive, neutral, and negative affective warning pictorials for using on cigarette labels. Implications on the affective warning pictorials design and validation.
\end{abstract}

\section{Introduction}

Warning pictorials are widely used on packaging and labelling of tobacco products or cigarette for antismoking campaigns in various countries such as Canada, Brazil, Singapore, and Thailand [1]-[3]. In order to satisfy the criteria of the World Health Organization Framework Convention on Tobacco Control (WHOFCTC), the warnings should be presented colorful graphics about health effect, hazards or emissions, and its size should be $50 \%$ or more of display areas, large, clear, visible, and legible [4]. While the goal of warning is to inform people in order to appreciate potential hazards [5], [6], to help reduce or eliminate risks [6]-[8], and attempt to promote safe behavior while reduce those unsafe behavior [6]. Thus, the design of warning pictorial is critical since it relates to the effectiveness of safety communication.

Several studies informed that current warning pictorials used on cigarette labels generated negative emotional responses. Mostly Canadian reported fear and disgust after viewing warning pictorial on cigarette label [1]. In Brazil, people described their emotional responses from warning pictorials of cigarette label on rating scales as unpleasant and moderately aroused [2]. While smoking people in Thailand also reported negative emotional responses, but still smoking [3]. Those people also reported that they attempted to avoid seeing warning pictorials on cigarette label by using their personal packaging cover, painting over warning pictorial, or importing cigarette from other countries [1]-[3].

There were some suggestions that warning pictorials used on cigarette label should not only focus on presenting the negative effect of smoking, but should provide positive information including benefits of quit smoking [9]. Azagba and Sharaf also suggested that the effectiveness of warning pictorials used on cigarette warning labels was mainly based on emotional responses and label presentation form [10]. Also, there was a study tried to improve the effectiveness of warning pictorials by introducing the idea of affective design of warning pictorials [11]. Affective design is defined as the design which capable to evoke appropriate affects in users [12]. In this paper, the term affect includes emotions, moods, and feelings.

Jiamsanguanwong and Umemuro examined the effect of positive, neutral, and negative affective states of people on their comprehension and hazard perception of standard industrial warning pictorials [13]. They manipulated affective states of participants using International Affective Pictures System (IAPS) [14] with an intention to introduce the concept of affective warning pictorial that pictorial itself could evoke target affective states on users. Result showed that people who were in positive affect condition perceived greater hazard from warning pictorials than those in neutral, and negative affect condition. The concept of affective warning pictorial should be validated in the context of cigarette label in order to confirm that some specific affective design of warning pictorial could improve the effectiveness of warning pictorial used on cigarette label. However, to the best of our knowledge, no one has design and validate the affective warning pictorial for using on cigarette label.

Thus, the purpose of present study were to design and validate affective warning pictorials for cigarette label. 
The results would provide three groups of affective warning pictorials as positive, neutral, and negative warning pictorials for using on cigarette label.

\section{Methods}

\subsection{Initial step}

The goal of this step is to find the possible warning pictorials used on cigarette label. First of all, ideas about the characteristics of warning pictorial used on cigarette label for anti-smoking campaign were collected by survey and brainstorming methods.

Fourteen Thai undergraduate and graduate engineering students participated in this brainstorming session. Five are males. They ranged in age from 21 to 26 years $(M=23.1, S D=1.9)$. Participants were asked to write down the ideas of possible warning pictorials used on cigarette label for anti-smoking campaign.

Thirty Thai smoking and non-smoking people participated in survey session. $56.7 \%$ are males. They ranged in age from 13 to 57 years $(M=29.5, S D=12)$. Participants were asked to draw or write down at least three ideas of possible warning pictorials used on cigarette label for anti-smoking campaign.

Total 198 ideas were collected and grouped into 5 groups; family, health, socials, finance, and others. Then, sixty warning pictorials were collected from free sources such as Thai health [15], and Tobacco Labelling Regulations [16], following the grouping ideas from brainstorming and survey session with cultural concerned. After that, all pictorials were primary equally classified into each positive, neutral, and negative warning pictorial groups for affective validation step.

\subsection{Affective validation}

This step is to validate affective warning pictorials from initial step. There were limited to a maximum of five participants per experimental session.

\subsection{Participants}

Sixty Thai undergraduate and graduate male engineering students participated in this study. They ranged in age from 20 to 28 years $(M=21.5, S D=1.69)$.

\subsection{Stimulus material}

\subsubsection{Affective picture stimuli}

Ten International Affective Picture System (IAPS) pictures were selected based on valence rating as neutral for males provided by manual of IAPS [14]. Their valence scores ranged from 4.05 to $5.19(M=4.56, S D=$ 1.54) and arousal scores ranged from 4.10 to $5.00(M=$ $4.56, S D=2.02$ ). The valence rating ranged from 1 : unpleasantness, to 9: pleasant, and arousal rating ranged from 1: calm, to 9: excited. For ethical reasons, pictures of mutilated and erotic imagery were excluded.

\subsubsection{Warning pictorials}

Sixty warning pictorials from the initial step were primary equally classified into positive, neutral, and negative pictorial groups. The majority of positive warning pictorials were from family group idea, neutral warning pictorials were from social and finance group idea, negative warning pictorials were from health group idea which are currently used on cigarette labels [16].

\subsection{Apparatus}

Affective picture stimulus and warning pictorials were presented by microsoft powerpoint and $4 \mathrm{~K}$ ultra HD, 65 inch television. The experiments were conducted in $6 \times 4$ $\mathrm{m}$ private laboratory room.

\subsection{Measurement}

The Self-Assessment Manikin (SAM) [17] was used to assess valence and arousal affect of participants (Figure 1). Affective valence ranges from 1: unpleasant to 9 : pleasant with 5 as neutral. Affective arousal ranges from 1: calm to 9: excited. Participants were asked to rate their affective states after affect manipulation and after viewing each of sixty warning pictorials.

\subsection{Procedure}

Prior to beginning, participants were asked to read and sign a consent form for their participation. Then, participants were given a brief instructions, the purpose of the research, and were informed the purpose of viewing the set of images was to manipulate their emotions.

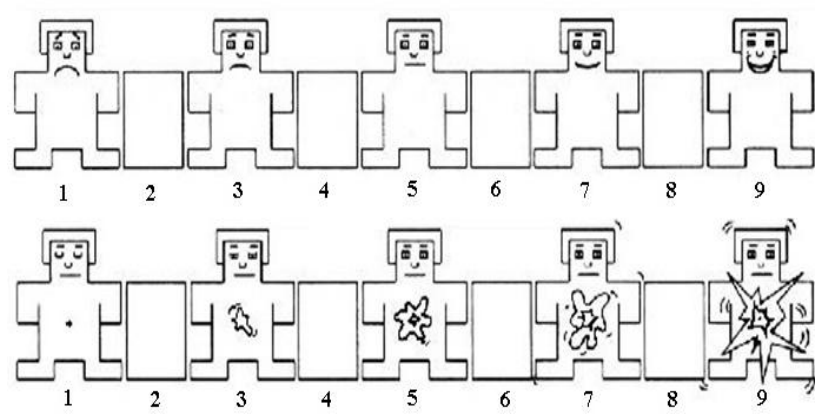

Figure 1. The Self-Assessment Manikin (SAM) [17]: valence (top panel), and arousal (bottom panel)

Ten neutral affective pictures were each presented for $6 \mathrm{~s}$, with a 2-s interval between pictures. After that, participants were asked to complete the Self-Assessment Manakin (SAM) questionnaire [14] to rate their present emotional valence and arousal (SAM I).

Then participants were given brief instructions and shown an example pictorial for practice. All sixty pictorials were randomly presented for $20 \mathrm{~s}$, with 10 -s interval between pictures. Participants were asked to immediately rate their emotional valence and arousal responses (SAM II) to each warning pictorials. 


\section{Results}

\subsection{Affect manipulation}

For the SAM I, sixty participants were achieved target neutral affective states. Their valence scores ranged from 3 to $6(M=4.50, S D=0.89)$ and arousal score ranged from 1 to $7(M=3.57, S D=1.70)$. This result indicated that all participants were successfully manipulated their emotion to neutral affective state.

\subsection{Affective validation}

Two participants were identified as outliers and excluded from the following analysis. Mean and standard deviation of valence and arousal scores for each group of affective warning pictorials were shown in table 1 .

One-way analysis of variance (ANOVA) were conducted with primary classified group of affective warning pictorial (positive, neutral, and negative) on the SAM II scores (valence, and arousal). The main effect of warning pictorial conditions were significant on valence score $(\mathrm{F}(2,3477)=3332.23, p<.001)$ and on arousal score $(\mathrm{F}(2,3477)=351.61, p<.001)$. Post hoc analyses revealed that participants perceived warning pictorials from positive group more positive as they rated higher valence rating scores than those warning pictorials from neutral group $(p<.001)$, or warning pictorials from negative group $(p<.001)$. Participants also rated higher

Table 1. Range of valence and arousal score of each warning pictorial groups. Before and after refining pictorials.

\begin{tabular}{|c|c|c|c|c|c|}
\hline \multicolumn{6}{|c|}{ Before refining pictorials } \\
\hline \multirow{2}{*}{$\begin{array}{l}\text { Warning } \\
\text { Pictorial } \\
\text { Groups }\end{array}$} & \multirow[b]{2}{*}{$\mathrm{N}$} & \multicolumn{3}{|c|}{ Valence score } & \multirow{2}{*}{$\frac{\text { Arousal score }}{\text { Min Mean (SD) }}$} \\
\hline & & Max Min & Mean (SD) & $\operatorname{Max}$ & \\
\hline Positive & 20 & 10 & & 22 & \\
\hline & 20 & $5.34 \quad 4.0$ & & 4.38 & \\
\hline & 20 & 3.641 .972 & $2.74(0.54)$ & 6.66 & 4.1 \\
\hline \multicolumn{6}{|c|}{ After refining pictorials } \\
\hline \multirow{2}{*}{$\begin{array}{l}\text { Warning } \\
\text { Pictorial } \\
\text { Groups }\end{array}$} & \multirow[b]{2}{*}{$\mathrm{N}$} & \multicolumn{2}{|c|}{ Valence score } & \multicolumn{2}{|r|}{ Arousal score } \\
\hline & & $\mathrm{Mi}$ & Mean (SD) & $\mathrm{M}$ & Min $\mathrm{Me}$ \\
\hline Positive & 10 & 7.216 .76 & 0.71 & 4.22 & 3.213 .8 \\
\hline & 10 & $4.94 \quad 4.45$ & 4.56 & 4.09 & $3.28 \quad 3.67$ \\
\hline Negative & 10 & $2.72 \quad 1.97$ & $2.28(0.25)$ & 6.66 & $5.57 \quad 6.20(0.42)$ \\
\hline
\end{tabular}

Valence score of warning pictorials from neutral group than those warning pictorials from negative group $(p<.001)$. While, warning pictorials from negative group had significantly higher arousal scores than those warning pictorials from positive group $(p<.001)$, or warning pictorials from neutral group $(p<.001)$. These results indicated that these affective warning pictorials successfully evoked target affective states on participants.

\subsection{Refining pictorials}

Ten out of twenties warning pictorials in each group were selected as final affective warning pictorial sets in order to reduce the variations of affective responses (SAM II) in each group (Figure 2). Mean and standard deviation of valence and arousal scores each group after refining the number of pictorials were shown in Table 1 . The criteria used for selecting the pictorials in each group based on their target valence score. For example, ten positive warning pictorials (Figure 2a) were selected by their top ten mean valence scores among other pictorials in positive group. Ten negative warning pictorials (Figure 2c) were selected by their least mean valence scores among other pictorials in negative group. Ten neutral warning pictorials (Figure $2 b$ ) were selected by the least mean absolute error from valence score of five as represented neutral rating score on SAM.

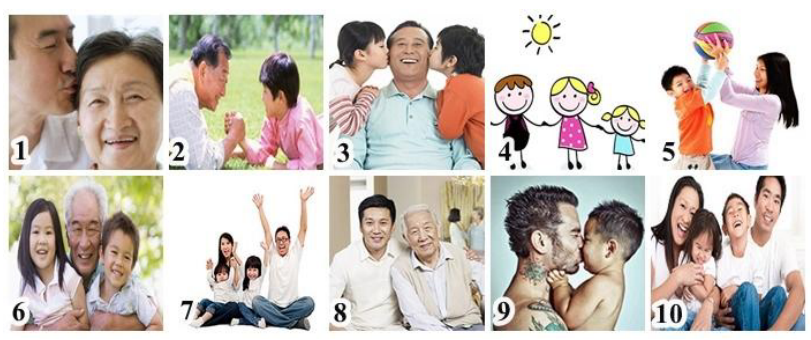

(a)

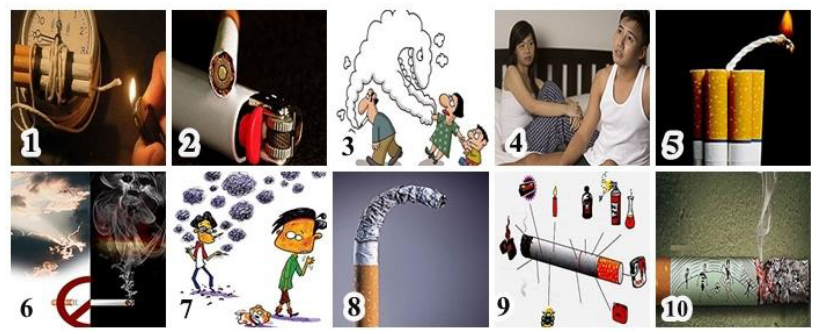

(b)

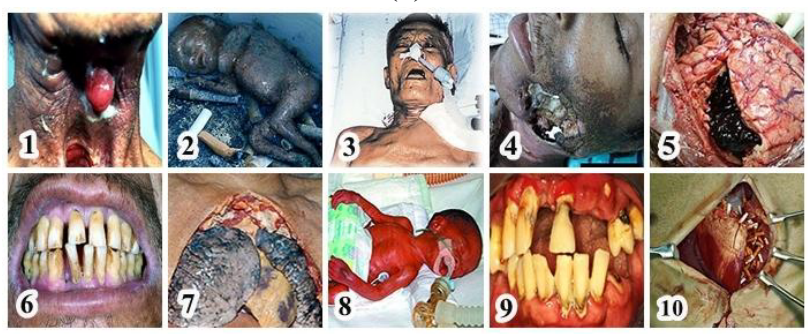

(c)

Figure 2. Final affective warning pictorials (a) positive warning pictorials (b) neutral warning pictorials (c) negative warning pictorials.

\section{Discussion}

Final affective warning pictorials were shown in Figure 2. The results confirmed its signiticantly evoked target affective states on participants to specific affective states such as positive, neutral, and negative affect. Ten neutral affective warning pictorials mainly presented the effect of smoking in terms of health, finance, and social (Figure $2 b)$. The pictorials used in this group had both real photos and cartoon pictorials. Positive warning

pictorials used in present study were majority pictorials of family reflecting the effect of smoking on their beloved people (Figure 2a). The pictorials of son kissing mother (Figure 2a-1) received highest rating valence scores among other pictorials $(M=7.21, S D=$ 
0.97). People may feel and reflect from the pictorial that he could be a good son with no smoking and takes care his mother's well-being. The positive rating responses from participants revealed that participants could be evoked positive affective states by this warning pictorials. While the least valence $(M=6.76, S D=1.08)$ and arousal $(M=3.21, S D=1.68)$ rating responses from participants among ten final set of pictorials was an only cartoon pictorial of family in this group (Figure 2a-4).

Negative warning pictorials in ten final pictorials were depicting health effect from smoking. These pictorials were actually the pictures used for reduce smoking campainge [15] such as chronic sore and cancer. The most negative pictorial which received the lowest valence rating scores $(M=1.97, S D=0.99)$ from participants was a pictorial of mouth cancer (Figure 2c-4). Moreover, participants rated their arousal scales $(M=$ $6.66, S D=1.77$ ) as highest for the pictorials (Figure 2c-2) in this negative group. Participants may feel frightened from the pictorials as they represented real human body of dreadful health effect from smoking.

After considered all the affective pictorials used in present study, cartoon-like pictorials evoked moderate affective responses on participants comparing to real images of people or cigarette. Although, there are one cartoon-like pictorials in final set of positive warning pictorial group, that pictorials were the least valence rating scores comparing other pictorials in same group. While there were no cartoon-like pictorial in negative warning pictorial group. The characteristics of pictorial (cartoon-like and real people pictorials) and its differences in people's affective responses should be investigated in future study. This may give some guideline for designing future affective pictorials.

\section{Conclusions}

This study aimed to design and validate affective warning pictorials used on cigarette label. Thirty affective warning pictorials (positive, neutral, and negative) were successfully validated as able to evoke specific target affective states on people.

There were limitations regarding to the scope of this study. The participants were limited to only male adults from engineering school. It is expected to have some different degree of affective responses regarding to the gender, culture, and generations in the results. Thus, it would be worth investigating with broader range of people from different generations, people with different background, and also female participants in future study. Moreover, future study should validate these affective warning pictorials in terms of warning effectiveness such as comprehension and hazard perception before real implementation.

\section{Acknowledgment}

This work was supported by Grants for Development of New Faculty Staff, Ratchadaphiseksomphot Endoment Fund, Chulalongkorn Univeristy, Bangkok, Thailand.

\section{References}

1. D. Hammond, G.T. Fong, P.W. McDonald, K.S. Brown, R. Cameron, AM J. Public Health, 94, 14421445 (2004)

2. B.E.M. Nascimento, L. Oliveira, A.S. Vieira, M. Joffily, S. Gleiser, M.G. Pereira, T. Cavalcante, E. Volchan, Tob Control, 17, 405-409 (2008)

3. S. Osiri, S. Kongkasawad, HSRI Journal, 1, 280-288 (2007)

4. WHO, Guidelines for implementation of the WHO Framework Convention on Tobacco Control, Article $11(2005)$

5. C.C. Braun, P.B. Mine, N.C.Silver, Int. J. Ind. Ergonom., 15, 179-187, (1995)

6. M.S. Wogalter, Ergodesigne USIHC, Rio de Janeiro: FAPERJ, 25-36 (2004)

7. M.F. Lesch, J. Safety Res., 34, 495-505 (2003)

8. M.F. Lesch, Accident Anal. Prev., 40, 1005-1012 (2008)

9. D. Sujirarat, P. Silpasuwan, C. Viwatwongkasem, N. Sirichothiratana, Southeast Asian J. Trop. Med. Public Health, 42, 134-141 (2011)

10. S. Azagba, M.F. Sharaf, Nicotine Tob Res., 15, 708717 (2013)

11. N. Schwarz, H. Bless, G. Bohner, Adv. Exp. Soc. Psychol., 24, 161-199 (1991)

12. H. Umemuro, Human-Computer Interaction, 5612, 683-692 (2009)

13. A. Jiamsanguanwong, H. Umemuro, Appl. Ergon., 45, 1362-1366 (2014)

14. P.J. Lang, M.M. Bradley, B.N. Cuthbert, International Affective Picture System (IAPS) NIMH Center for the Study of Emotion and Attention, 3958 (1997)

15. P. Vartesatokkit, Luckpim Publishing, Warning Pictorial on Cigarette, (2009)

16. Tobacco Labelling Resource Centre. Thailand. (2013) Retrieved September 20, 2015, from http://www.tobaccolabels.ca/countries/thailand

17. M.M. Bradley, P.J. Lang, J. Behav. Ther. Exp. Psy., 25, 49-59 (1994) 